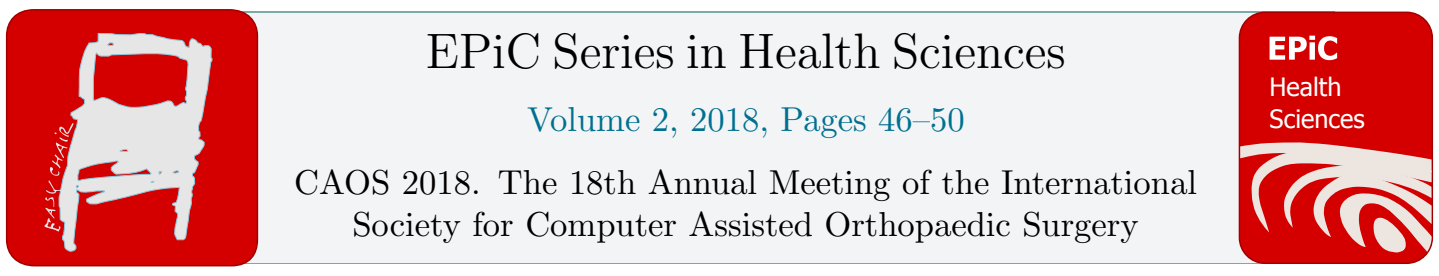

\title{
A deep learning-based approach for localization of pedicle regions in preoperative CT scans
}

\author{
Hooman Esfandiari ${ }^{1,2 *}$, Carolyn Anglin ${ }^{3}$, Pierre Guy ${ }^{4}$, Antony J Hodgson ${ }^{1,2}$ \\ ${ }^{1}$ University of British Columbia, Vancouver, Canada \\ ${ }^{3}$ University of Calgary, Calgary, Canada \\ ${ }^{2}$ Surgical Technologies Laboratory, UBC, Vancouver, Canada \\ ${ }^{4}$ Department of Orthopaedic Surgery, UBC, Vancouver, Canada \\ hooman.esfandiari@ubc.ca
}

\begin{abstract}
Pedicle screw fixation is a common yet technically demanding procedure. Due to the proximity of the inserted implant to the spinal column, a malplaced screw can cause neurological injury and subsequent postoperative complications. A common surgical routine starts with preoperative volumetric image acquisition (e.g. computed tomography) based on which the surgeons can highlight the planned trajectory. This process is generally done manually, which is error prone and time consuming.

The primary purpose of this paper is to develop an automatic pedicle region localization based on preoperative CTs. This system can automatically annotate the CT scans to identify the regions corresponding to the pedicles and thus provide important information about the anatomical placement of the CT scan that can be useful for intraoperative implant position assessment (e.g. to initialize the 2D-3D registration). On the other hand, the pedicle localization can be exploited for preoperative planning.

We designed and evaluated a fully convolutional neural network for the task of pedicle localization. A large training, validation and testing datasets $(5000,1000,1000$ images separately) were created using a custom data augmentation process that could generate unique vertebral morphologies for each image. After evaluation on the validation and test data, the Dice similarity coefficients between the pedicle regions detected by the trained network and the ground truth was 0.85 and 0.83 respectively.

The proposed deep-learning-based algorithm was capable of automatically localizing the regions corresponding to the pedicles based on the preoperative CT scans. Therefore, a reliable initial guess for the 2D-3D registration process needed for intraoperative implant position assessment can be achieved. This system also has potential use in automating the preoperative planning.
\end{abstract}

\footnotetext{
* Corresponding Author
} 


\section{Introduction}

First introduced in 1944, pedicle screw insertion is still the most popular surgical practice for spinal fusion. Although there are substantial benefits, pedicle screw insertion remains a technically demanding procedure in close proximity to the spinal cord and blood vessels. A malplaced pedicle screw can lead to serious postoperative complications, reported in about $6 \%$ of patients (Nevzati, 2014).

A number of different surgical procedures exist for pedicle screw fixation, ranging from freehand methods (Allam 2013) to more advanced computer-assisted navigation and robotic approaches (Patil 2012). Spinal fusion surgery normally begins by acquiring a volumetric image of the vertebrae of interest. This preoperative image is then used to highlight the insertion trajectory as part of preoperative planning. If a computer-assisted procedure is used, the preoperative image is also used for intraoperative surgical navigation.

The predominant features of interest in the preoperative images are the pedicle regions in each vertebrae, because of the acute proximity to the spinal column and because most implant perforations occur medially or laterally to the pedicles; even experienced surgeons can malplace up to $5 \%$ of the screws medially and up to $15 \%$ inferolaterally (Mirza 2003).

Current preoperative surgical practice usually involves the surgeon visually and manually interpreting the preoperative images to determine the desired insertion trajectory. This process is mainly qualitative in nature and error-prone, and there is thus a need for an automated pedicle region localization system.

On the other hand, when intraoperative implant position assessment is concerned (Esfandiari, 2016), the location of the pedicle regions in the acquired volumetric images can provide crucial information to initialize the $2 \mathrm{D}-3 \mathrm{D}$ registration of the preoperative and intraoperative images.

Therefore, an automatic pedicle localization method can benefit the intraoperative implant position assessment (by providing reliable initialization for the 2D-3D registration process) as well as the preoperative planning.

Early work on pedicle segmentation dates back at least as far as 2004, when researchers presented an analytical model for pedicle segmentation and patient-specific implant size planning (Wicker 2004). However, this paper lacked any experimental results and was not tested on clinical data. In 2011, a more sophisticated approach was introduced that was based on morphological representation of each vertebrae (Lee, 2011). While achieving satisfactory results for the lumbar spine $(2.11 \pm 0.17$ $\mathrm{mm}$ ), this method required precise segmentation of the preoperative CT scans in order to reconstruct $3 \mathrm{D}$ models of each vertebrae. In addition to this, it required comparatively long computation times (on the order of 6 minutes), which would be an obstacle to clinical adoption of such a system.

Accordingly, the purpose of this study is to develop and evaluate a fast, accurate and automated pedicle localization algorithm for processing CT volumes that can be easily adopted in a real surgery.

\section{Materials and Methods}

We hypothesized that a convolutional neural network trained for semantic segmentation purposes could potentially be exploited to localize the pedicle regions in preoperative volumetric images in a fully automatic manner. In particular, we used a proprietary implementation of Fully Convolutional Neural-networks (FCNs; Long 2015) that could be trained in an end-to-end fashion. The network takes volumetric images of vertebrae as input and, after running the data through a number of convolutional and deconvolutional blocks, produces a binary mask highlighting the pixels belonging to the pedicle regions (Figure 1). 


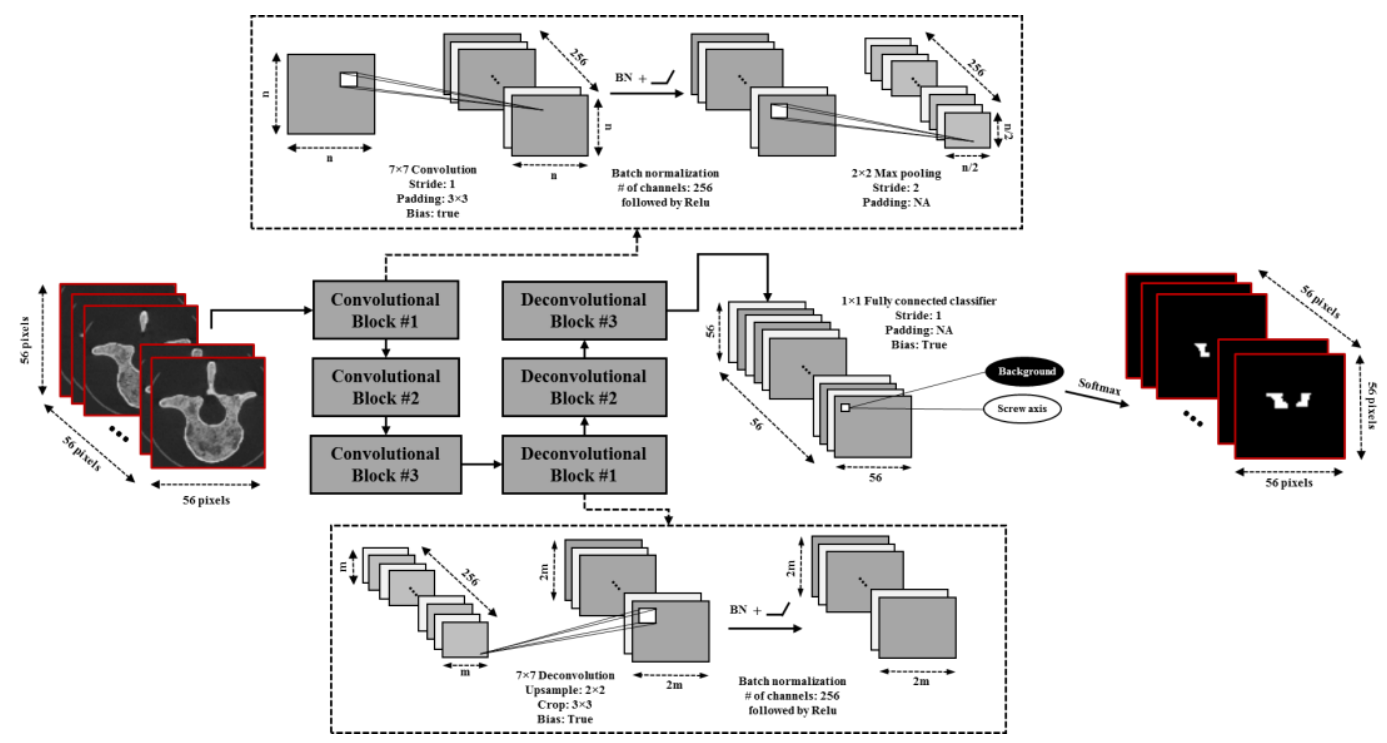

Figure 1: The schematic diagram of the network's architecture.

It is often difficult or expensive to acquire a large number of clinical volumesets. Therefore, we designed a data augmentation process that could generate a large number of unique training instances based on a small number of manually segmented volumetric images (Figure 2). Six synthetic lumbar bone models with realistic radiographic properties (Ammolite Biomodels, Calgary, Canada) were scanned with a CT machine (HR-PQCT, Scanco-XTREME CT) to generate the required volumetric images. The images were then manually annotated using the MITK-GEM software (Pauchard 2016) to identify the voxels belonging to the pedicle regions (i.e. to generate the ground truth masks). The CT scans, together with their corresponding pedicle masks, were then plugged into a BSpline deformable transformation module that used a number of varying parameters (grid size, deformation field distribution and deformation magnitude) as well as an affine transformation in a controlled random fashion to generate a large number of augmented image/mask sets, each having a unique morphology (5000 sets for training and 1000 sets for validation).

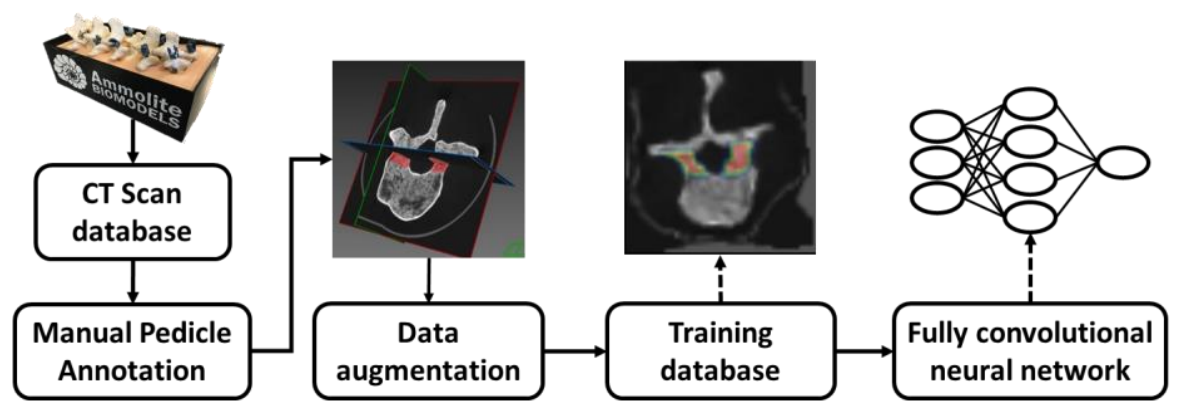

Figure 2: Overall pipeline of the pedicle localization system.

The data augmentation code was written using the SimpleITK library in Python environment. The implementation of the code required for designing and training the machine learner was done using the Keras library with Tensorflow backend in a Python environment. The computing platform used 
for the data augmentation and FCN training was an Intel Core i7 CPU $(2.60 \mathrm{GHz})$ processor with 16 GB of RAM and an NVIDIA Geforce GTX 960M GPU.

After completing the training process and in order to verify the performance of the model, two Dice similarity coefficients were calculated to estimate the overlap between the reference and estimated pedicle regions - one based on the validation dataset and one based on an unseen testing dataset (between the predicted pedicle regions and the ground truth masks). The unseen testing dataset was generated using a similar data augmentation routine based on a separate CT scan that was not involved in the training process (1000 test sets).

\section{Results}

Generation of the training, validation and testing datasets took 40 minutes and training the deep learner took 6 hours on the aforementioned computing platform. The Dice similarity coefficients for the validation and testing datasets were calculated as 0.85 and 0.83 , respectively (with no post processing). The maximum inference (i.e. prediction) time of the trained network was less than one second.

\section{Discussion}

The large Dice coefficients found in this study suggest that the deep-learning-based automatic algorithm was always successful in identifying the pedicle regions in the validation and testing CT volumesets. The discrepancies in the overlapped region estimates were comparatively minor, with edge errors typically on the order of $1 \mathrm{~mm}$. No preprocessing of the CT volumes (e.g. segmentation of bone surfaces and surface reconstruction) was needed. The current system, however was only trained and tested on CT imagery and can only handle one vertebra at a time (as opposed to a fully connected spinal column that would be present in live surgeries), so future work would aim to address this issue.

By being able to automatically identify the pedicle areas in a spinal CT image, this algorithm can provide a reliable initial guess for the 2D-3D registration process we are using in our intraoperative position assessment system. This algorithm also has potential uses in simplifying and speeding the identification and localization of key anatomical structures in surgery planning processes.

\section{Disclosure}

No relevant disclosures.

\section{Acknowledgements}

This work has been supported by the Canadian Natural Sciences and Engineering Research Council (NSERC) and the Canadian Institute of Health Research (CIHR). We thank the Centre for Hip Health and Mobility for providing the lab facilities used in this study and the Institute for Computing, Information and Cognitive Systems for program support. 


\section{References}

1 - Nevzati E, Marbacher S, Soleman J, et al (2014) Accuracy of Pedicle Screw Placement in the Thoracic and Lumbosacral Spine Using a Conventional Intraoperative Fluoroscopy-Guided Technique: A National Neurosurgical Education and Training Center Analysis of 1236 Consecutive Screws. World Neurosurgery 82:866-871.

2 - Allam Y, Silbermann J, Riese F, Greiner-Perth R (2013) Computer Tomography Assessment of Pedicle Screw Placement in Thoracic Spine: Comparison between Free Hand and a Generic 3Dbased Navigation Techniques. Eur Spine J 22:648-653.

3 - Patil S, Lindley EM, Burger EL, Yoshihara H, Patel VV (2012) Pedicle Screw Placement With O-arm and Stealth Navigation. Orthopedics 35(1): e61-65.

4 - Mirza SK, Wiggins GC, Kuntz Iv C, et al (2003) Accuracy of Thoracic Vertebral Body Screw Placement Using Standard Fluoroscopy, Fluoroscopic Image Guidance, and Computed Tomographic Image Guidance: a Cadaver Study. Spine 28:402-413

5 - Esfandiari H, Newell R, Bernard R, Anglin C, Street J, Swamy G, Hodgson AJ (2016) An Intraoperative (Post-Placement) Pedicle Screw Position Assessment System. In: 16th Annual Meeting of the International Society of Computer Assisted Orthopaedic Surgery, Osaka, Japan.

6 - Wicker R, Tedla B (2004) Automatic Determination of Pedicle Screw Size, Length, and Trajectory from Patient Data. In: Engineering in Medicine and Biology Society, 2004. IEMBS'04. 26th Annual International Conference of the IEEE. IEEE, p 1487-1490

7 - Lee J, Kim S, Kim YS, et al (2011) Automated Surgical Planning and Evaluation Algorithm for Spinal Fusion Surgery with Three-dimensional Pedicle Model. In: Intelligent Robots and Systems (IROS), 2011 IEEE/RSJ International Conference on. IEEE, pp 2524-2531

8 - Long J, Shelhamer E, Darrell T (2015) Fully Convolutional Networks for Semantic Segmentation. In: Proceedings of the IEEE Conference on Computer Vision and Pattern Recognition. pp 3431-3440

9 - Pauchard Y, Fitze T, Browarnik D, et al (2016) Interactive Graph-cut segmentation for Fast Creation of Finite Element Models from Clinical CT Data for Hip Fracture Prediction. Computer Methods in Biomechanics and Biomedical Engineering 19:1693-1703. 